\title{
EVALUACIÓN COLABORATIVA PARA LA ENSEÑANZA DE LAS CIENCIAS SOCIALES EN EDUCACIÓN PRIMARIA
}

Collaborative evaluation for teaching Social Sciences in Primary Education.

Avaliação colaborativa para o ensino das Ciências Sociais na Educação Primária.

María Pilar Molina Torres (1)

(1) Universidad de Córdoba, España. Teléfono: +34 957212559. Correo electrónico: pilar.molina@uco.es

\section{Resumen}

El trabajo cooperativo es una herramienta que a menudo crea sinergias didácticas para la evaluación del proceso de enseñanza-aprendizaje. En este trabajo se presenta una experiencia educativa que tiene como objetivo general la propuesta de varios instrumentos de evaluación necesarios para trabajar las ciencias sociales en Educación Primaria. El método utilizado se adecua al trabajo colaborativo y a la construcción del conocimiento mediante la dinámica de grupos de investigación que nos permiten una mayor optimización de los recursos empleados. Los resultados mostraron al alumnado de sexto de Primaria implicado en el aprendizaje experimental y siempre desde una perspectiva que implique que se está trabajando con algo que resulta divertido, haciéndolos responsables de dinamizar lo máximo posible el uso del portafolio, los dosieres de prácticas, la autoevaluación con rúbricas, el folio giratorio.

Palabras clave: Aprendizaje por descubrimiento; ciencias sociales; competencias educativas; evaluación

\begin{abstract}
Cooperative work is a tool that often creates didactic synergies for the evaluation of the teaching-learning process. This paper presents an educational experience that has as a general objective the proposal of several assessment instruments necessary to work in the social sciences in Primary Education. The method used is adapted to collaborative work and to the construction of knowledge through the dynamics of research groups that allow us to optimize the resources used. The results showed the sixth grade students involved in experimental learning and always from a perspective that implies that they
\end{abstract}


are working with something that is fun, making them responsible for making the use of the portfolio as much as possible, the dossiers of practices, the self-assessment with rubrics, rotating sheet.

Keywords: Learning by discovery; social sciences; educational competences; assessment

\section{Resumo}

O trabalho cooperativo é uma ferramenta que muitas vezes cria sinergias didáticas para a avaliação do processo de ensino-aprendizagem. Este trabalho apresenta uma experiência de ensino que tem como objetivo geral a apresentação de vários instrumentos de avaliação necessários para atuar nas ciências sociais na Educação Primária. O método utilizado adequa-se ao trabalho colaborativo e à construção do conhecimento mediante a dinâmica dos grupos de investigação, os quais permitem otimizar os recursos utilizados. Os resultados mostraram que os alunos do sexto ano estavam envolvidos na aprendizagem experimental e sempre numa perspetiva de trabalharem com algo que é divertido, tornando-os responsáveis por dinamizar tanto quanto possível, o uso do portefólio, dos dossiers de práticas, a autoavaliação com rubricas, folha rotativa.

Palavras-chave: Aprendizagem pela descoberta; ciências sociais; competências educativas; avaliação

\section{Introducción}

En la enseñanza de las ciencias sociales la construcción de los conceptos sociales es un proceso esencial que diferencia claramente el conocimiento social de otros tipos de conocimientos (Cuenca, 2014). Es más, en la enseñanza del patrimonio histórico es necesario que la evaluación de las estrategias implementadas permita detectar tanto las fortalezas como las debilidades para mejorar la calidad del aprendizaje. A continuación, exponemos un recorrido evaluativo sobre la gestión cooperativa del aula que se fundamentará en aprender colaborativamente a partir del trabajo común (Zariquiey, 2016). 


\section{Contextualización}

La experiencia se realiza con un grupo de veintiséis alumnos y alumnas de sexto de Educación Primaria, de los cuales 17 son niños y 9 son niñas. Esta distribución que no significó ningún impedimento en relación con el aprendizaje de los/as estudiantes, sí lo supuso en cuanto a los agrupamientos, pues tienden a unirse con los de su mismo género. La asignatura para el desarrollo de la propuesta fue ciencias sociales, de modo que pudimos contextualizarlos en el patrimonio cultural de nuestra ciudad, Córdoba, en época romana, y reconocer en ese patrimonio heredado una identidad histórica (Molina, 2018).

\section{Diseño y desarrollo}

El método de enseñanza utilizado en el desarrollo y diseño de la experiencia estuvo adaptado a las características de los alumnos que componen el grupo-clase. Para evitar las clases magistrales, se proponen una serie de talleres didácticos centrados en el aprendizaje por descubrimiento para que el alumnado investigue sobre la civilización romana. Los agrupamientos fueron flexibles de manera que pudieran trabajar en gran grupo, pequeños grupos y por parejas, fomentando con ello el trabajo cooperativo, el ajuste de los ritmos y espacios, la variedad de recursos (Figura 1).

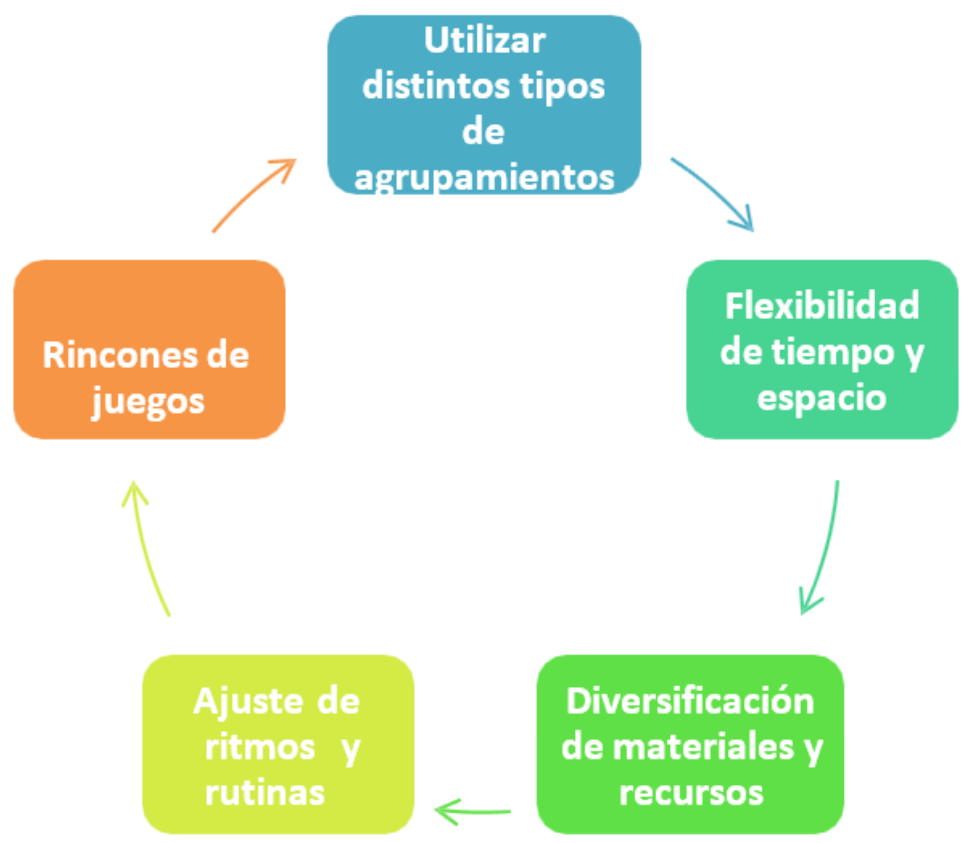

Figura 1.

Mapa conceptual con estrategias de trabajo grupales. Fuente: elaboración propia. 
El proyecto se estructura en tres fases:

- Fase de investigación (1 sesión). Mediante la utilización del folio giratorio recogemos las respuestas a varias preguntas que planteamos: ¿Qué sabemos? ¿Qué queremos saber? ¿Cómo lo vamos a lograr?

- Fase de implementación (5 sesiones). En cada taller se plantearon actividades de aprendizaje visual que versarán sobre la familia romana, el urbanismo, las creencias y la economía de la colonia.

- Fase de síntesis y recapitulación de ideas (2 sesiones). Como actividad final cada grupo realizó una presentación en Genially con los resultados de su investigación y la temática asignada. Por último, valoramos el proyecto a través de rúbricas de evaluación.

\section{Evaluación}

Para la propuesta hemos tenido en cuenta dos tipos de evaluación continua. Por un lado, tenemos la heteroevaluación que realiza el profesor a los alumnos en cada uno de los talleres, con una escala de valoración donde se tienen en cuenta contenidos conceptuales, procedimentales y actitudinales (Tabla 1). Los valores de esta escala son $\mathrm{A}, \mathrm{B}, \mathrm{C}$ o D; donde A sería el valor más positivo y, por el contrario, $\mathrm{D}$ el más negativo (Tabla 1).

Tabla 1.

Escala de valoración del profesor hacia los alumnos y recuento de las respuestas

\begin{tabular}{|c|c|c|c|c|}
\hline Alumno/a & & & & \\
\hline Aspectos que valorar & $\mathbf{A}$ & B & $\mathrm{C}$ & D \\
\hline $\begin{array}{l}\text { Es capaz de contestar a las preguntas sobre el tema encaminadas a la meta de este } \\
\text { trabajo. }\end{array}$ & 22 & 4 & 0 & 0 \\
\hline Conoce cuál era el motor principal de la economía de la Córdoba romana. & 20 & 5 & 1 & 0 \\
\hline Reconoce los principales edificios de la ciudad y sus funciones. & 19 & 5 & 1 & 1 \\
\hline Va avanzando en el camino hacia un aprendizaje significativo. & 25 & 1 & 0 & 0 \\
\hline $\begin{array}{l}\text { Maneja herramientas para investigar y darle respuesta a las distintas cuestiones } \\
\text { sobre el tema que se trabaja. }\end{array}$ & 23 & 2 & 1 & 0 \\
\hline Comprende y responde a las preguntas acerca de lo que está trabajando. & 18 & 7 & 1 & 0 \\
\hline $\begin{array}{l}\text { Ayuda, respeta, colabora con sus compañeros. Mantiene una actitud correcta } \\
\text { durante las clases. }\end{array}$ & 21 & 5 & 0 & 0 \\
\hline Muestra una actitud positiva e interés por aprender durante la propuesta. & 25 & 1 & 0 & 0 \\
\hline
\end{tabular}

Por otra parte, contamos con la autoevaluación de los alumnos y alumnas durante los talleres realizados. En dicha evaluación se tuvieron en cuenta sus propias 
actuaciones, las de los compañeros y los recursos utilizados. Todo ello fue recogido en una escala de valoración en la que se incluye un apartado de comentarios individual (Tabla 2).

Tabla 2.

Escala de valoración de los alumnos y recuento de las respuestas

\begin{tabular}{|c|c|c|c|}
\hline Mi actuación & Sí & A veces & No \\
\hline He aportado algunas ideas al grupo & 25 & 0 & 1 \\
\hline He ayudado a realizar las tareas & 24 & 2 & 0 \\
\hline He prestado atención a las indicaciones de la maestra & 21 & 4 & 1 \\
\hline He participado en las actividades & 25 & 1 & 0 \\
\hline Me he aburrido en clase & 8 & 2 & 16 \\
\hline Me he implicado en el proyecto. & 22 & 1 & 3 \\
\hline \multicolumn{4}{|l|}{ Comentarios } \\
\hline Los talleres & Sí & & \\
\hline El trabajo me ha interesado & 26 & & \\
\hline He conseguido aprender & 24 & & \\
\hline Esperaba algo diferente & 4 & & \\
\hline Me gustaría cambiar algo & 3 & & \\
\hline
\end{tabular}

\section{Conclusiones}

El proceso de enseñanza aprendizaje reflejado en estas líneas proporciona un feedback entre alumnado y docente para identificar los problemas que vayan surgiendo durante el aprendizaje, así como posibles soluciones. Se facilitó la interacción y el aprendizaje colaborativo para hacer este intercambio de ideas grupales. Del mismo modo, estas estrategias didácticas promovieron la responsabilidad individual y grupal en el proceso, la capacidad de transmisión de ideas, las habilidades sociales, la autoevaluación y el trabajo en equipo (Johnson \& Johnson, 2014). En definitiva, y atendiendo al recuento de respuestas de las escalas de valoración, podemos confirmar la implicación de los/as estudiantes en una metodología de indagación con la que no están acostumbrados a trabajar, ni tampoco a evaluar.

\section{Referencias}

Cuenca, J. M. (2014). El papel del patrimonio en los centros educativos: hacia la socialización patrimonial. Tejuelo, 19, 76-96. 
Johnson, D. W., \& Johnson, R. T. (2014). La evaluación en el aprendizaje cooperativo: cómo mejorar la evaluación individual a través del grupo. Madrid: SM.

Molina, M. P. (2018). Aprendizaje basado en proyectos ABP para la enseñanza de la Historia. En A. I. Ponce y J. Ortuño (Eds), Pensando el patrimonio: Usos y recursos en el ámbito educativo (pp. 127-138). Murcia: Universidad de Murcia

Zariquiey, F. (2016). Cooperar Para Aprender. Transformar el aula en una red de aprendizaje cooperativo. Madrid: SM. 